\title{
Conductive Sub-micrometric Wires of Platinum-Carbonyl Clusters Fabricated by Soft-lithography
}

\author{
Pierpaolo Greco $^{1}$, Massimiliano Cavallini ${ }^{1}$, Pablo Stoliar ${ }^{1}$, Santiago David \\ Quiroga ${ }^{1}$, Soumya Dutta ${ }^{1}$, Stefano Zacchini ${ }^{2}$, Maria Carmela Iapalucci ${ }^{2}$, Vittorio \\ Morandi, Silvia Milita ${ }^{3}$ Pier Giorgio Merli ${ }^{3}$ and Fabio Biscarini ${ }^{1}$. \\ 1- CNR-ISMN Bologna, Via P. Gobetti 101, I-40129 Bologna, Italy. \\ 2- Dipartimento di Chimica Fisica e Inorganica, Università di Bologna, V.le \\ Risorgimento 4, 40136 Bologna, Italy \\ 3- CNR-IMM Bologna, Via P. Gobetti 101, I-40129 Bologna, Italy.
}

\section{Experimental}

Materials: The $\left[\mathrm{NBu}_{4}\right]_{2}\left[\mathrm{Pt}_{15}(\mathrm{CO})_{30}\right]$ solutions employed (typically $0.6 \mathrm{~g} / \mathrm{L}$ ) were prepared by dissolving $\left[\mathrm{NBu}_{4}\right]_{2}\left[\mathrm{Pt}_{15}(\mathrm{CO})_{30}\right]$ (prepared according to the literature [15]) in Dimethylformamide (DMF) (Aldridch spectroscopic grade quality) or tetrahydrofurane (THF) (Aldridch spectroscopic grade quality).

Substrates: The substrates consist in a piece $10 \times 10 \mathrm{~mm}^{2}$ of commercial glass for optical microscopy. It was cleaned using the standard protocol: sonication for two minutes in electronic-grade water (milli-pure quality), two minutes in acetone (Aldridch chromatography quality) then two minutes in 2-propanol (Aldridch spectroscopic grade quality).

Films preparation: The films were prepared by drop casting $20 \mu 1$ of the $\left[\mathrm{NBu}_{4}\right]_{2}\left[\mathrm{Pt}_{15}(\mathrm{CO})_{30}\right]$ solution on glass under nitrogen or in air. Before measure, they were left 24 hours at room temperature in order to have a complete solvent evaporation.

Stamp preparation: The elastomeric polydimethylsiloxane (Sylgard 184 Down Corning) stamps were prepared by replica molding of a blank Digital Versatile Disk support that acts as a structured master. The curing process was carried for 6 hours at $60^{\circ} \mathrm{C}$. Once cured, the replica is peeled off from the master and washed in pure ethanol for one hour. The stamp motif consists of parallel lines with a periodicity of $740 \mathrm{~nm}$, width at half height $410 \mathrm{~nm}$ and $100 \mathrm{~nm}$ deep.

Optical Microscopy: Optical micrographs were recorded with a Nikon i-80 microscope equipped with epi-illuminator and cross polars. 
Atomic Force Microscopy: AFM images were recorded with a commercial AFM (Thermomicroscope) operating in air, in contact mode $\left(25^{\circ}\right.$ with relative humidity $55 \%$ ). $\mathrm{Si}_{3} \mathrm{~N}_{4}$ cantilevers (Nanosensors, Wetzlar-Blankenfeld), with typical curvature radius of a tip $10 \mathrm{~nm}$ were used. All images are unfiltered. The topographic images were corrected line-by-line for background trend effects by removal of the secondorder polynomial fitting. Image analysis was carried out ex-situ with image analysis software IMAGE-SXM 1.79 and analysis of the autocorrelation spectra and power spectral density were carried out with Igor Pro 4.0.

Finite Element Modeling: Finite element calculations were performed with software Comsol Multiphysics 3.2.

Electrical Measurements: The electrical property of the $\mu$-stripes was measured using two probe techniques. Two gold pads (50 nm thick) were thermally evaporated on top of the series of $\mu$-stripes through a square TEM grid (50 mesh) (SPI Supplies, Brand TEM Grid), to be used as electrodes (see fig. 8a). The current-voltage characteristics were carried out using Keithley sourcemeter $(6430$ subfemtoammeter). The length of the Pt-stripes was measured using optical microscopy and the width and the thickness of the Pt-stripes were measured analyzing the topography of the sample by AFM.

Scanning Electron Microscopy: The Scanning Electron Microscopy (SEM) images were obtained with a ZEISS 1530 SEM operating at $20 \mathrm{keV}$. The instrument was equipped with detectors for Backscattered Electrons (BSE) and Secondary Electrons (SE) imaging and with an Energy Dispersive X-Ray Spectrometer (EDX) for X-Rays microanalysis.

$X$ Ray Diffraction: XRD measurements have made by means a MZVI-Seifert diffractometer. A focus line X-ray beam, produced by a conventional $\mathrm{Cu} \mathrm{K} \alpha$ tube powered at $40 \mathrm{kV}$ and $50 \mathrm{~mA}$, was collimated (divergence in the diffraction plane $0.025^{\circ}$ ) by a parabolic graded multilayer (W/Si) mirror by OSMIC (model GO18A). A slit of $0.1 \mathrm{~mm}$ before the sample acts to limit the lateral size of the beam while Soller slits before the detector reduces the acceptance divergence of the scintillator detector to $0.4^{\circ}$. 